\title{
3 Research Square

\section{Clinical Course and Risk Factors for severe Disease and Death of Adult Critically III Inpatients with COVID-19 in Toulouse, France: A Retrospective Cohort Study}

\section{William Buffières}

Critical Care Unit. University Hospital of Purpan. Toulouse

\section{Benjamine Sarton}

Critical Care Unit. University Hospital of Purpan. Toulouse

Charline Zadro

Radiology Department. University Hospital of Rangueil. Toulouse

\section{Fanny Vardon Bounes}

Critical Care Unit. University Hospital of Rangueil. Toulouse

\section{Vincent Minville}

Critical Care Unit. University Hospital of Purpan. Toulouse

\section{Bernard Georges}

Critical Care Unit. University Hospital of Rangueil. Toulouse

\section{Thierry Seguin}

Critical Care Unit. University Hospital of Rangueil. Toulouse

\section{Jean Marie Conil}

Critical Care Unit. University Hospital of Rangueil. Toulouse

\section{Stephanie Ruiz}

Critical Care Unit. University Hospital of Rangueil. Toulouse

\section{Samia Collot}

Radiology Departement. University Hospital of Rangueil. Toulouse

\section{Guillaume Ducos}

Critical Care Unit. University Hospital of Purpan. Toulouse

\section{Marie Virtos}

Critical Care Unit. University Hospital of Purpan. Toulouse

\section{Diane Osinski}

Neurocritical Care Unit

\section{David Rousset}

Neurocritical Care Unit. University Hospital of Purpan. Toulouse

Thomas Geeraerts

Neurocritical Care Unit. University Hospital of Purpan. Toulouse 


\section{Thomas Filleron}

Epidemiology Departement. Claudius-Regaud Institute, IUCT-Oncopole, Toulouse

\section{Benoit Bataille}

Critical Care Unit. Narbonne Hospital. Narbonne

Jacques Izopet

Virology Department. University Hospital of Purpan. Toulouse

Jean Ruiz

Critical Care Unit. Claudius-Regaud Institute, IUCT-Oncopole. Toulouse

\section{Veronique Ramonda}

Critical Care Unit. University Hospital of Purpan. Toulouse

\section{Olivier Fourcade}

Neurocritical Care Unit. University Hospital of Purpan. Toulouse

\section{Beatrice Riu}

Critical Care Unit. University Hospital of Purpan. Toulouse

\section{Damien Guinault}

Critical Care Unit. University Hospital of Purpan. Toulouse

\section{Stein Silva ( $\nabla$ silvastein@me.com )}

University Teaching Hospital of Purpan

\section{Research}

Keywords: COVID-19, comorbidities, hypertension, Kidney Disease Improving Global Outcomes (KDIGO)

Posted Date: September 4th, 2020

DOl: https://doi.org/10.21203/rs.3.rs-66770/v1

License: (c) (i) This work is licensed under a Creative Commons Attribution 4.0 International License. Read Full License 


\section{Abstract}

\section{Background}

To explore risk factors for unfavorable outcome (death or requiring invasive mechanical ventilation at 28 days from ICU admission) of critically ill COVID-19 patients hospitalized in the Toulouse region

\section{Methods}

Retrospective cohort of critically ill COVID-19 patients sequentially admitted to 12 ICUs in Toulouse region (March 9, 2020, to April 8, 2020). All patients had laboratory confirmed SARS-CoV-2 infection and required invasive mechanical ventilation. Baseline characteristics, pathophysiological respiratory data, clinical outcomes, viral shredding, and chest CT scan were collected.

\section{Results}

A total of 150 patients were included (median age, 68 years (interquartile range, (IQR), 58-72; 81\% male). The most common comorbidities were hypertension (77, 51\%) and obesity (42, 28\%). At ICU admission, the median $\mathrm{PaO} 2 / \mathrm{FiO} 2$ ratio was 138 (IQR,112-178). During hospitalization, the rate of ventilator-acquired pneumonia (VAP) was 61\% and 51 (34\%) patients had acute kidney injury (AKI) with a Kidney Disease Improving Global Outcomes (KDIGO) score > 1. The 28-day mortality was $15.3 \%$, and 50 (33\%) patients had unfavorable outcome. We found that VAP $(5.91 ; 2.10-10.03 ; p$ value $=0.002)$ and AKI with a KDIGO score $>1(4.71 ; 1.69-14.41 ; p$ value $=0.004)$ were associated with increased odds of unfavorable outcome. Neither, chest CT scan data on admission, nor pathophysiological respiratory data during ICU stay were associated to patient's outcome.

\section{Conclusion}

The potential risk factors of AKI and VAP could help clinicians to identify patients with poor prognosis at an early stage. Targeted care of these factors might have a significant impact on COVID-19 patient's outcome.

\section{Introduction}

As the global outbreak of coronavirus disease 2019 (Covid-19), caused by severe acute respiratory syndrome coronavirus 2 (SARS-CoV-2), is rapidly evolving, its full spectrum of effects is becoming evident - from self-limiting respiratory tract illness to severe acute respiratory distress syndrome (ARDS), multiple organ failure and death. Since initial detection of the virus, more than 6000000 cases of Covid19 have been confirmed worldwide, with the first reported cases in France on January 24, 2020. On April 7, 2020, there had been 1860 laboratory-confirmed Covid-19 cases in Toulouse, the fourth-largest city in France. Among them, 183 patients were critically ill. The clinical spectrum of SARS-CoV-2 appears to be wide, with the most severe cases corresponding to severe viral pneumonia with respiratory failure and even death. Previous studies have only described the general epidemiological findings, clinical 
presentation and outcomes of patients of SARS-CoV-2 pneumonia ${ }^{1-4}$. Nevertheless, specific information characterizing critically ill SARS-CoV-2 patients remains scarce, despite its high importance to reduce mortality. Although some case series have been published ${ }^{5-7}$, no previous studies have been done among critically ill Covid-19 patients with definite outcomes. Additionally, there is no evidence regarding the prognostic value of information that might be collected at intensive care unit (ICU) admission and throughout ICU stay. In particular, we clearly lack data about the effects on outcome of extrapulmonary organs failures, that might be observed in severe forms of SARS-CoV-2 infection.

Here, we present details of all patients admitted to 12 ICUs from Toulouse city and region, with critically ill laboratory confirmed Covid-19 patients and definite clinical outcome (death or requirement of invasive mechanical ventilation at 28 days from ICU admission) as of April 8, 2020. We aim to explore baseline and follow-up risk factors for unfavorable outcome and describe the clinical course of symptoms, radiological findings, viral shedding, and temporal changes of laboratory findings during ICU hospitalization.

\section{Methods}

\section{Study design and participants}

This retrospective observational study was performed at the University Teaching Hospital of Toulouse, which was the regional coordinating center for the "Covid-19 Toulouse ICU Network". All consecutive patients with laboratory-confirmed SARS-CoV-2 infection referred to one of the ICUs among 12 hospitals in the network between March 9 and April 8, 2020, were enrolled. Critically ill patients were defined as those admitted to the ICU who required mechanical ventilation and had a fraction of inspired oxygen (FiO2) of at least $60 \%$ or more 7 . Identification of critically ill patients was achieved by reviewing and analyzing available electronic medical resources.

\section{Data collection}

We reviewed clinical electronic medical records, nursing records, laboratory findings, and radiological examination for all recruited patients. The admission data of these patients were collected. Data were evaluated and collected, by using a case record form from the standardized WHO/International Severe Acute Respiratory and Emerging Infection Consortium case report forms. Any missing or uncertain records were collected and clarified through direct communication with involved health-care providers. Epidemiological, demographic, clinical, laboratory, radiological, treatment and outcome data were extracted. All data were checked by two physicians (WB and DG) and a third researcher (SS) adjudicated any difference in interpretation between the two primary reviewers.

\section{Laboratory procedures}

Laboratory confirmation of SARS-CoV2 infection was performed by collecting naso-pharyngeal swab, sputum, and bronchoalveolar lavage fluids. The SARS-CoV2 genome was detected using a multiplex RT- 
PCR assay targeting two regions within the RNA-dependent RNA polymerase gene and the Pather-Fusion systems ${ }^{8}$. Routine blood examinations were serum biochemical tests (including renal and liver function, creatine kinase, lactate dehydrogenase, and electrolytes), myocardial enzymes, serum ferritin, complete blood count and coagulation profiles. CT scan or chest radiographs were also done inpatients. Frequency of examinations was determined by the treating physician ${ }^{9}$.

\section{Radiological procedures}

Thoracic CT scan images were acquired in all patients. 100 chest CT scans were performed between $24 \mathrm{~h}$ before and $24 \mathrm{~h}$ after patient's ICU admission. All CT scan were obtained in the supine position. Images were reconstructed with a slice thickness of $1 \mathrm{~mm}$ and an interval of $1 \mathrm{~mm}$, respectively. Intravenous contrast agents were used in only 22 cases. Reconstructed images were transmitted to the workstation and picture archiving and communication systems (PACS) of Toulouse University Hospital for multiplanar reconstruction pos-processing. Images were analyzed by two radiologists (CZ and SC, both senior thoracic radiologists with more than 15 years' experience). All Digital Imaging and Communication in Medicine (DICOM) images from the CT studies were analyzed without access to clinical or laboratory findings. The evaluators independently and freely assessed the CT features using both axial CT images and multiplanar reconstruction images. After separate evaluations, any disagreements were resolved by discussion and consensus. CT scan Covid-19 related features were recorded following previously described criteria ${ }^{10}$.

We used two radiological scores specifically designed for Covid-19 use. First, the Chest Computed Tomography Severity Score (CT-SS) ${ }^{11}$ was defined by summing up individual score from 20 lung regions; scores of 0,1 , and 2 were respectively assigned for each region if parenchymal opacification involved $0 \%$, less than $50 \%$, or equal than $50 \%$ of each region (theoretical range of CT-SS from 0 to 40 ). Second, the Total Severity Score (TSS) ${ }^{12}$ was based on summing up the acute lung lesion involving each lung lobe (overall 5 lobes), which was scored as 0 (0\%), 1 (1-25\%), 2 (26-50\%), $3(51-75 \%)$, or $4(76-100 \%)$, respectively. The TSS was reached by summing the 5 lobes scores (theoretical range of TSS from 0 to 20).

\section{Definitions}

Sepsis and septic shock were defined according to the 2016 Third International Consensus Definition for Sepsis and Septic Shock. Secondary infection was diagnosed when patients showed symptoms or signs of pneumonia or bacteremia and a positive culture of a new pathogen was obtained from lower respiratory tract specimens (i.e. sputum, endotracheal aspirate, or bronchoalveolar fluid) or blood samples after admission. Ventilator- associated pneumonia was diagnosed according to the guidelines for treatment of hospital-acquired and ventilator- associated pneumonia ${ }^{13}$. Acute kidney injury was diagnosed according to the KDIGO clinical practice guidelines ${ }^{14}$ and acute cardiac injury was diagnosed if serum levels of cardiac biomarkers (e.g. high-sensitivity cardiac troponin I) were above the 99th percentile upper reference limit or new abnormalities were shown in electrocardiography or echocardiography ${ }^{15}$. Acute Cor pulmonale was defined by echocardiography as septal dyskinesia with a 
dilated right ventricle (end-diastolic RV/left ventricle $(\mathrm{LV} \text { ) area ratio }>0,6)^{16}$. Coagulopathy was defined as a 3-second extension of activated prothrombin time or a 5-second extension of activated partial thromboplastin time.

\section{Statistical analysis}

Continuous and categorical variables were presented as median (IQR) and $n(\%)$, respectively. We used the Mann Whitney $\mathrm{U}$ test, $\mathrm{X}^{2}$ test, or Fisher's exact test to compare differences between where appropriate. The composite criterion was used as outcome assessment main criteria: unfavorable (death or requiring mechanical ventilation 28 days after ICU admission), favorable (alive and free from invasive mechanical ventilation 28 days after ICU admission). To explore the risk factors associated with outcome, univariable and multivariable logistic regression models were used. Considering the total number of deaths $(n=23)$ in our study and to avoid overfitting in the model, five variables were chosen for multivariable analysis based on previous reports and clinical constraints.

Previous studies have reported older age ( $>65$ years) associated with adverse clinical outcomes in adults SARS-CoV-2 and Middle East respiratory syndrome (MERS) ${ }^{17}$. There are recent reports of adults infected with SARS-CoV-2 who have developed an acute kidney injury (as assessed by the Kidney Disease Improving Global Outcomes, KDIGO score) ${ }^{18}$, and patients with previous kidney disease (as assessed by Chronic Kidney Disease, CKD score) $)^{19}$ appears to be highly vulnerable to Covid-19. Previous studies have shown that hemodynamic shock (defined by the need of use of a vasopressor drug to maintain mean arterial pressure $>65 \mathrm{mmHg}$ ) is a marker of severity in critically ill patients. Patients with Acute Respiratory Distress Syndrome (ARDS) are more likely to develop ventilator- associated pneumonia. In ARDS patients, VAP is associated with an increased length of stay and perhaps an additional mortality 20 . Recent reports highlight the possibility of an increases risk of ventilator- associated pneumonia in Covid19 cases related to immunological factors ${ }^{21}$. Therefore, we chose age, previous kidney disease (CKD), acute kidney injury (KDIGO), hemodynamic shock (use vasopressor support during the first three days after ICU admission) and ventilator- associated pneumonia as the five variables for our multivariate logistic regression model. We excluded variables from the univariate analysis if their between-group differences were not significant, if their accuracy was unconfirmed and if the number of events was too small to calculate odds ratios.

A two-sides alpha of less than 0.05 was considered statistically significant. Statistical analyses were done using the SPSS (IBM SPSS Statistics for Windows, Version 24.0. Armonk, NY: IBM Corp.) software.

\section{Results}

995 adult patients were hospitalized in Toulouse and region with Covid-19 before April 8, 2020. Among them, we included 150 critically ill inpatients (Fig. 1). 23 (15,3\%) patients died during ICU stay and 106 $(70,7 \%)$ were discharged. 50 have an unfavorable outcome (death or requiring invasive mechanical ventilation at 28 day after ICU admission). The median age of the 150 patients was 68 years (IQR 58- 
72), ranging from 19 to 88 years, and most patients were male (Table 1). Comorbidities were present in nearly half of patients, with hypertension being the most common comorbidity followed by obesity (BMI $>30 \mathrm{~kg} / \mathrm{m}^{2}$ ) and diabetes. Renin-Angiotensin-Aldosterone System (RAAS) inhibitors were present as home medication in almost half of patients. On admission, SOFA score was 6 (IQR 4-8) and 15\% of patients have extra-pulmonary organ failure (Table 1). Most of the patients were in severe ARDS according to Berlin definition (PaO2/ FiO2 was 138 (IQR 112-178). Lymphopenia and thrombopenia occurred in 128 (85\%) and 41 (27\%) of cases, respectively. Chest CT scan on ICU admission shown severe lung anomalies according to CT-SS and TSS scores (26, IQR 22-30 and 12, IQR 10-15, respectively). 142 (95\%) patients received antibiotics and $120(80 \%)$ received antivirals (Table 1$).$ 
Table 1

Demographic, clinical, laboratory and radiographic findings of ICU patients on ICU admission. Data are

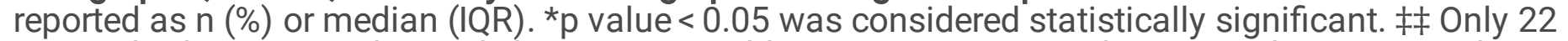
patients had contrast-enhanced chest CT scan Abbreviations: BMI = Body mass index; $C O P D=$ Chronic Obstructive pulmonary disease; RAAS = Renin-Angiotensin-Aldosterone System; ACE-I = Angiotensinconverting enzyme inhibitor; $A R B s=$ Angiotensin II receptor blockers; NSAID = non-steroidal antiinflammatory drugs; SOFA = Sequential Organ Failure Assessment score; SAPS II = Simplified Acute

Physiology Score, version $2 ; \mathrm{FiO} 2=$ Fraction of inspired oxygen; $\mathrm{PaO} 2=$ partial pressure of arterial oxygen; PEEP = Positive end-expiratory pressure; CT-SS = Computed Tomography Severity Score; TSS = Total severity score.

\begin{tabular}{|c|c|c|c|c|}
\hline & $\begin{array}{l}\text { All } \\
n=150\end{array}$ & $\begin{array}{l}\text { Favourable } \\
n=100\end{array}$ & $\begin{array}{l}\text { Unfavourable } \\
n=50\end{array}$ & $\begin{array}{l}p \\
\text { value }\end{array}$ \\
\hline Demographics & $68(58-72)$ & $65(56-72)$ & $70(66-73)$ & 0.004 \\
\hline Age, median (IQR), years & $121(80.7)$ & $79(79)$ & $42(84)$ & 0.46 \\
\hline Male sex - no. (\%) & $\begin{array}{l}27.3(25.5- \\
30.5)\end{array}$ & $\begin{array}{l}27.3(25.4- \\
30.5)\end{array}$ & $\begin{array}{l}27.7(25.4- \\
30.3)\end{array}$ & 0.89 \\
\hline BMI, median (IQR) & $42(28)$ & $27(27)$ & $15(30)$ & 0.92 \\
\hline Co-morbidities - no. (\%) & $77(51)$ & $48(48)$ & $29(58)$ & 0.25 \\
\hline Obesity (BMI $\geq 30 \mathrm{~kg} / \mathrm{m}^{2}$ ) & $32(21)$ & $23(23)$ & $9(18)$ & 0.48 \\
\hline пурелтель⿻ы & $40(27)$ & $21(21)$ & $19(38)$ & 0.027 \\
\hline Dianeles & $16(11)$ & $7(7)$ & $9(18)$ & 0.04 \\
\hline Dysiाpiciema & $18(12)$ & $9(9)$ & $9(18)$ & \\
\hline Coronary artery disease & $14(9)$ & $6(6)$ & $8(16)$ & 0.015 \\
\hline COPD & $3(2)$ & $3(3)$ & 0 & 0.55 \\
\hline Cnronic Kianey alsease & $9(6)$ & $4(4)$ & $5(10)$ & 0.16 \\
\hline 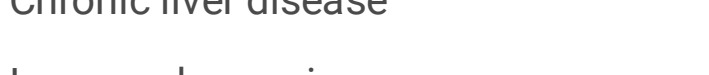 & $13(9)$ & $10(10)$ & $3(6)$ & 0.54 \\
\hline 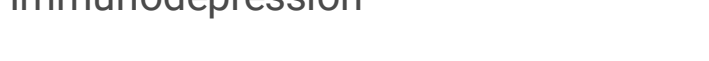 & $58(39)$ & $39(39)$ & $19(38)$ & 0.91 \\
\hline & $6(4)$ & $5(5)$ & $1(2)$ & 0.66 \\
\hline Home medications - no. (\%) & $11(7)$ & 7 (7) & $4(8)$ & 1.00 \\
\hline RAAS inhibitors (ACE-I or ARBs) & $9(7-11)$ & $10(7-11)$ & $9(6-10)$ & 0.06 \\
\hline NSAID & $15(10)$ & $9(9)$ & $6(12)$ & 0.56 \\
\hline 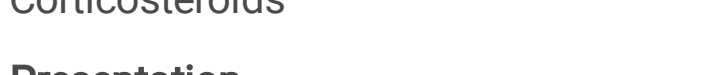 & $4(3)$ & $1(1)$ & $3(6)$ & 11 \\
\hline riesentialion & $22(15)$ & $8(8)$ & $14(28)$ & 0.047 \\
\hline $\begin{array}{l}\text { Median symptom onset to intubation } \\
\text { (IQR), d }\end{array}$ & $6(4-8)$ & $6(4-7)$ & $7(6-8)$ & 0.0001 \\
\hline Coinfection on ICU admission & $37(31-43)$ & $34(29-41)$ & $40(37-44)$ & $\stackrel{<}{<.0001}$ \\
\hline
\end{tabular}




\begin{tabular}{|c|c|c|c|c|}
\hline & 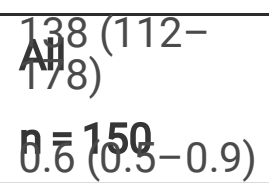 & 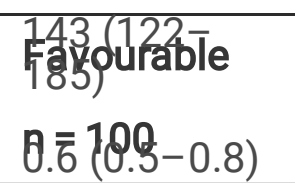 & 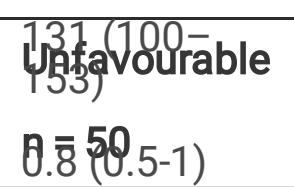 & $\begin{array}{l}\text { p.014 } \\
\text { yaluse }\end{array}$ \\
\hline Shock on ICU admission & $10(8-12)$ & $10(8-12)$ & $10(9-12)$ & 0.99 \\
\hline $\begin{array}{l}\text { Non-respiratory acute organ failure - } \\
\text { no. (\%) }\end{array}$ & $6.5(6.2-6.9)$ & $6.5(6.3-6.8)$ & $6.5(6.2-7.1)$ & 0.82 \\
\hline $\begin{array}{l}\text { SOFA score on admission, median } \\
\text { (IQR) }\end{array}$ & $\begin{array}{l}22(20-23) \\
11(10-13)\end{array}$ & $21(20-23)$ & $\begin{array}{l}22(20-24) \\
11(10-13)\end{array}$ & 0.55 \\
\hline SAPS II, median (IQR) & $40(34-50)$ & $39.8(35-50)$ & $40.5(33-49)$ & 0.70 \\
\hline $\begin{array}{l}\text { Respiratory parameters on admission, } \\
\text { median (IQR) }\end{array}$ & $0.8(0.6-1)$ & $0.8(0.6-1.1)$ & $0.7(0.5-0.9)$ & 0.057 \\
\hline $\mathrm{PaO}_{2}: \mathrm{FiO}_{2}$ ratio ${ }^{* *}$ & $\begin{array}{l}197(147- \\
270)\end{array}$ & $\begin{array}{l}219(159- \\
308)\end{array}$ & $\begin{array}{l}167(136- \\
225)\end{array}$ & 0.85 \\
\hline $\mathrm{FiO}_{2}^{* \star}$ & $\begin{array}{l}1495(870- \\
2145)\end{array}$ & $\begin{array}{l}1340(870- \\
2740)\end{array}$ & $\begin{array}{l}1790(955- \\
2010)\end{array}$ & 0.0006 \\
\hline PEEP $\left(\mathrm{cm} \mathrm{H}_{2} 0\right)^{\star \star \star}$ & $69(58-89)$ & $65(56-80)$ & $77(66-118)$ & 00045 \\
\hline Tidal volume $\left(\mathrm{mL} \cdot \mathrm{kg}^{-1}\right.$ of PBW) ${ }^{* \star}$ & $8.8(6-13)$ & $8.8(6-12)$ & $9(6-15)$ & 0.47 \\
\hline Plateau pressure $\left(\mathrm{cm} \mathrm{H}_{2} \mathrm{O}\right) \neq$ & $14(9-36)$ & $13(8-20)$ & $24(12-39)$ & 0.058 \\
\hline Driving pressure $\left(\mathrm{cm} \mathrm{H}_{2} \mathrm{O}\right) \ddagger$ & 144 (93- & $138(80-207)$ & $1.4(1.1-1.7)$ & 0.96 \\
\hline Static compliance $\left(\mathrm{mL}\right.$ per $\left.\mathrm{cm} \mathrm{H}_{2} \mathrm{O}\right) \neq$ & 215) & $1757(1090-$ & & 0.65 \\
\hline $\begin{array}{l}\text { Laboratory findings on admission, } \\
\text { median (IQR) }\end{array}$ & $\begin{array}{l}1851(996- \\
3208)\end{array}$ & $\begin{array}{l}2942) \\
438(338-\end{array}$ & $\begin{array}{l}1853(808- \\
3486)\end{array}$ & $\begin{array}{l}0.69 \\
0.72\end{array}$ \\
\hline Lymphocyte count, $\times 10^{9} / \mathrm{L}$ & $\begin{array}{l}441(344- \\
618)\end{array}$ & $619)$ & $\begin{array}{l}443(374- \\
572)\end{array}$ & 0.55 \\
\hline Platelet count, $\times 10^{9} / \mathrm{L}$ & $24(21-29)$ & $5(5-5)$ & $25(22-28)$ & 0.73 \\
\hline D-Dimer, mg/L & $5(5-5)$ & $26(22-30)$ & $5(5-5)$ & NA \\
\hline Creatinine, mol/L & $26(22-30)$ & $12(9-15)$ & $27(23-30)$ & \\
\hline Total bilirubin, mol/L & $12(10-15)$ & $0(0)$ & $12(10-15)$ & \\
\hline Troponin I, ng/L & $1(0,67)$ & & $1(2)$ & \\
\hline Lactate, mmol/L & & & & \\
\hline C-reactive protein, mg/L & & & & \\
\hline Ferritin, $g / L$ & & & & \\
\hline Lactate dehydrogenase, $\mathrm{U} / \mathrm{L}$ & & & & \\
\hline Serum albumin, $g / L$ & & & & \\
\hline
\end{tabular}




\begin{tabular}{|lllll|}
\hline & All & Favourable & Unfavourable & p \\
& $n=150$ & $n=100$ & $n=50$ & value \\
\hline $\begin{array}{l}\text { Computed tomography findings on } \\
\text { admission }\end{array}$ & & & \\
Number of lobes affected, median (IQR) & & & \\
CT-SS, median (IQR) & & & \\
TSS, median (IQR) & & & \\
$\begin{array}{l}\text { Pulmonary embolism on admission- } \\
\text { no. (\%) fł }\end{array}$ & & & \\
\hline
\end{tabular}

The median time from illness onset to ICU discharge was 20 days (IQR 11-31), whereas the median time to death was 13 days (IQR 10-20); (Table 2). 136 (90.7\%) patients required mechanical ventilation for a median duration of 14 days (IQR 8-24). The median time from illness onset to invasive mechanical ventilation was 9 days (IQR 7-11). Neuromuscular blockers agents and prone positioning were used in 127 (85\%) and 97 (65\%), respectively. Vasopressors were frequently required after ICU admission (127 patients, $85 \%) .51$ (34\%) patients have criteria of acute kidney injury and $13(25 \%)$ of them have renal replacement therapy. A ventilator- associated pneumonia occurred in $91(60,7 \%)$ of patients requiring invasive mechanical ventilation (Table 2). The frequency of complications was higher in survivors than in non survivors. 
Table 2

Treatment and outcomes. Data is reported as $n(\%) .{ }^{*} p$ value $<0.05$ was considered statistically significant. Abbreviations: $\mathrm{ECMO}=$ extracorporeal membrane oxygenation; $\mathrm{MV}=$ mechanical ventilation; PS = Pressure support mode; PCR = Polymerase chain reaction; VAP = ventilator-associated pneumonia;

SOFA = Sequential Organ Failure Assessment score.

\begin{tabular}{|c|c|c|c|c|}
\hline & $\begin{array}{l}\text { All } \\
n=150\end{array}$ & $\begin{array}{l}\text { Favourable } \\
n=100\end{array}$ & $\begin{array}{l}\text { Unfavourable } \\
n=50\end{array}$ & $\begin{array}{l}p \\
\text { value }\end{array}$ \\
\hline Treatments - no. (\%) & $127(85)$ & $78(78)$ & $49(98)$ & \multirow{2}{*}{$\begin{array}{l}< \\
0.0001\end{array}$} \\
\hline Neuromuscular blockers & $97(65)$ & $57(57)$ & $40(80)$ & \\
\hline Prone position & $17(11)$ & $5(5)$ & $12(24)$ & 0.0001 \\
\hline Inhaled pulmonary vasodilator & $5(3)$ & $1(1)$ & $4(8)$ & 0.0005 \\
\hline ECMO & $97(87)$ & $58(58)$ & $39(78)$ & 0.11 \\
\hline Vasopressors & $13(9)$ & $3(3)$ & $10(20)$ & 0.06 \\
\hline Renal Replacement Therapy & $120(80)$ & $85(85)$ & $35(70)$ & 0.001 \\
\hline Antiviral therapy & $96(64)$ & $71(71)$ & $25(50)$ & 0.10 \\
\hline Hydroxychloroquine & $28(19)$ & $16(16)$ & $12(24)$ & 0.012 \\
\hline Lopinavir/Ritonavir & $9(6)$ & $8(8)$ & $1(2)$ & 0.24 \\
\hline Remdesivir & $142(95)$ & $94(94)$ & $48(96)$ & 0.27 \\
\hline Antibiotics & $59(39)$ & $31(31)$ & $28(56)$ & 0.78 \\
\hline Corticosteroids & $14(8-24)$ & $14(8-19)$ & $30(13-39)$ & 0.0031 \\
\hline Outcomes & $9(5-14)$ & $9(5-12)$ & $15(9-21)$ & \multirow{2}{*}{$\dot{0} 0001$} \\
\hline Duration of invasive MV, median (IQR), d & $5(2-9)$ & $5(3-7)$ & $9(0-21)$ & \\
\hline Volume-controlled mode & $11(6-18)$ & $11(7-15)$ & $19(12-26)$ & 0.0001 \\
\hline PS mode & $6(2-10)$ & $4(2-8)$ & $10(6-15)$ & \multirow{2}{*}{$\begin{array}{l}< \\
0.0001\end{array}$} \\
\hline Number of days of, median (IQR): & $1(0-3)$ & $1(0-2)$ & $2(1-5)$ & \\
\hline Sedation & $18(12)$ & $10(10)$ & $8(16)$ & 0.0001 \\
\hline Neuromuscular blockade & $33(22)$ & $8(8)$ & $25(50)$ & 0.6196 \\
\hline Prone position & $19(15-24)$ & $17(14-23)$ & $22(17-26)$ & \multirow{2}{*}{$\begin{array}{l}< \\
0.0001\end{array}$} \\
\hline Extubation failure-no. (\%) & $26(21-33)$ & $24(21-31)$ & $30(25-35)$ & \\
\hline Tracheostomy-no. (\%) & $99(66)$ & $57(57)$ & $42(84)$ & \multirow{2}{*}{$\begin{array}{l}0.034 \\
0.012\end{array}$} \\
\hline Duration of viral shedding, median (IQR), $d$ & $91(60.7)$ & $50(50)$ & $41(82)$ & \\
\hline $\begin{array}{l}\text { Time from 1st positive PCR to 1st negative } \\
\text { PCR }\end{array}$ & $19(13)$ & $8(8)$ & $11(22)$ & 0.0002 \\
\hline
\end{tabular}




\begin{tabular}{|c|c|c|c|c|}
\hline & $\begin{array}{l}\text { Ali } \\
n \subseteq(5) \\
=(450\end{array}$ & $\begin{array}{l}3(3) \\
\text { Favourable } \\
3(3) \\
h=100\end{array}$ & $\begin{array}{l}\text { Onfavourable } \\
3(6)=50 \\
n=5\end{array}$ & $\begin{array}{l}0.015 \\
\text { p.lye }\end{array}$ \\
\hline \multirow{2}{*}{$\begin{array}{l}\text { Time from symptoms onset to } 1 \text { st negative } \\
\text { PCR }\end{array}$} & $4(3-6)$ & $3(2-5)$ & $6(3-7)$ & \multirow{2}{*}{$<.0001$} \\
\hline & $-2(-4 ; 0)$ & $-2(-4 ; 0)$ & $-1(-4 ; 1)$ & \\
\hline Secondary infection & $5(2-8)$ & $4(1-7)$ & $10(5-15)$ & 0.26 \\
\hline VAP & $6(4)$ & $2(2)$ & $4(8)$ & $<.0001$ \\
\hline Bacteremia & $51(34)$ & $22(22)$ & $29(58)$ & \multirow{3}{*}{$\begin{array}{l}0.096 \\
< \\
0.0001\end{array}$} \\
\hline Invasive fungal infection & $22(14.7)$ & $13(13)$ & $9(18)$ & \\
\hline Aspergillosis & $12(8)$ & $4(4)$ & $8(16)$ & \\
\hline SOFA score at Day 7 , median (IQR) & $17(11)$ & $5(5)$ & $12(24)$ & 0.085 \\
\hline$\Delta$-SOFA (from baseline to Day 7 ) & $49(33)$ & $28(28)$ & $21(42)$ & \multirow{3}{*}{$\begin{array}{l}0.18 \\
0.21 \\
0.008\end{array}$} \\
\hline \multirow{2}{*}{$\begin{array}{l}\text { Median duration of vasopressor support } \\
(\mathrm{IQR}), \mathrm{d}\end{array}$} & $38(25)$ & $22(22)$ & $16(32)$ & \\
\hline & $23(15)$ & $13(13)$ & $10(20)$ & \\
\hline Acute cardiac injury - no. (\%) & $25(17)$ & $11(11)$ & $14(28)$ & \multirow{2}{*}{$\begin{array}{l}0.0004 \\
0.0052\end{array}$} \\
\hline Acute kidney injury & $14(9)$ & $3(3)$ & $11(22)$ & \\
\hline Stage 1 & $\begin{array}{l}35.5(29- \\
42)\end{array}$ & $36(31-43)$ & $34(13-41)$ & \multirow{12}{*}{$\begin{array}{l}< \\
0.0001 \\
< \\
0.0001\end{array}$} \\
\hline Stage 2 & & $19(11-26)$ & $32(13-40)$ & \\
\hline Stage 3 & $20(11-31)$ & $27(19-34)$ & $35(13-41)$ & \\
\hline Thromboembolic events & $27(18-37)$ & $11(11)$ & $22(44)$ & \\
\hline Pulmonary embolism & $33(22)$ & $1(1)$ & $20(40)$ & \\
\hline Deep venous thrombosis & $21(14)$ & 0 & $23(46)$ & \\
\hline Major bleeding & $23(15,3)$ & & & \\
\hline \multicolumn{4}{|l|}{ Acute cor pulmonale } & \\
\hline \multicolumn{4}{|l|}{ Median days follow-up (IQR) } & \\
\hline \multicolumn{4}{|l|}{ ICU length of stay, median (IQR), d } & \\
\hline \multicolumn{4}{|l|}{ Hospital length of stay, median (IQR), d } & \\
\hline $\begin{array}{l}\text { Still in hospital at the end of follow-up - no. } \\
(\%)\end{array}$ & & & & \\
\hline
\end{tabular}

Still in ICU at the end of follow-up - no. (\%)

Death before Day 28 
In univariable analysis of ICU admission data, odds of unfavorable outcome were higher in patients with most severe ARDS (lower Pa02/FiO2). Platelet count, creatinine and troponin I were also associated with unfavorable outcome. Regarding univariable analysis of data collected during ICU stay, the duration of mechanical ventilation, sedation, neuromuscular blockade, were associated to unfavorable outcome (Table 2). Interestingly, the duration of viral shedding was also related to patient's outcome. Secondary infection, encompassing bacteremia and ventilator-associated pneumonia had also a significant impact over patient outcome. Finally, extra-pulmonary organ failures, including acute kidney injury were associated to 28-day outcome.

We included 150 patients with complete data for all variables (100 favorable and 50 unfavorable outcomes). We found that chronic kidney disease at admission, ventilator- associated pneumonia and acute kidney injury were associated with increased odds of unfavorable outcome (Table 3). Patients survival probability was significantly related to acute kidney injury severity (Fig. 2). The median duration of viral shedding was 24 (IQR 21-31) and 30 days (IQR 25-35) from illness onset in patients with favorable and unfavorable outcome, respectively (e-Figure 1). It is worth noting, that the clinical course and the duration of invasive mechanical ventilation, sedation and pharmacological neuromuscular blocking, were different between patients with favorable and unfavorable outcome (Table 2; e-Figure 1). Nevertheless, in these patients requiring invasive mechanical ventilation, no significative differences were observed between the respiratory parameters that were recorded after patient's admission during the ICU stay (e-Figure 2). Finally, chest CT scan standardized scores that were assessed at ICU admission, were not associated to patients 28 days outcome (e-Table 1 ). 
Table 3

Risks factors associated with unfavorable outcome. Unfavorable outcome was defined as death or requiring invasive mechanical ventilation at 28 days from ICU admission. $p$ value $<0.05$ was considered statistically significant. Abbreviations: SAPS II = Simplified Acute Physiology Score, CKD = Chronic Kidney Disease, SOFA = Sequential Organ Failure Assessment score. $\mathrm{Cl}=$ confidence interval.

\begin{tabular}{|c|c|c|c|c|}
\hline & $\begin{array}{l}\text { Univariable OR } \\
\left.\text { [Cl }{ }_{95 \%}\right]\end{array}$ & $\begin{array}{l}p \\
\text { value }\end{array}$ & $\begin{array}{l}\text { Mutivariable OR } \\
{\left[\mathrm{Cl}_{95 \%}\right]}\end{array}$ & $\begin{array}{l}p \\
\text { value }\end{array}$ \\
\hline Risk factors at baseline & 4.69 [1.94-13.18] & 0.001 & \multirow{2}{*}{$\begin{array}{l}2.45[0.90- \\
7.50]\end{array}$} & 0.092 \\
\hline \multirow{2}{*}{ Age $\geq 60$ years } & $2.92[1.02-8.68]$ & 0.047 & & - \\
\hline & $2.31[1.09-4.89]$ & 0.028 & & - \\
\hline Coronary artery disease & 6.16 [1.69-29.18] & 0.010 & \multirow{3}{*}{$\begin{array}{l}4.68[1.12- \\
24.57]\end{array}$} & 0.046 \\
\hline Dyslipidemia & $1.63[1.33-2.05]$ & & & - \\
\hline Chronic Kidney Disease & $1.11[1.06-1.17]$ & 0.001 & & 0.054 \\
\hline SOFA score on admission & 0.99 [0.99-1.00] & $\begin{array}{l}<.001 \\
0.001\end{array}$ & \multirow{2}{*}{$\begin{array}{l}1.06 \text { [1.006- } \\
1.123]\end{array}$} & - \\
\hline SAPS II & $2.55[1.20-5.44]$ & 0.041 & & - \\
\hline $\mathrm{PaO}_{2}: \mathrm{FiO}_{2}$ ratio on admission & $1.02[1.01-1.03]$ & 0.014 & & - \\
\hline Low platelet count on admission & $1.01[1.00-1.02]$ & 0.002 & & - \\
\hline Serum Creatinine & $0.41[0.20-0.82]$ & 0.153 & & - \\
\hline Troponin I & $2.83[1.41-5.77]$ & 0.013 & & - \\
\hline Risk factors during follow-up & $1.07[1.01-1.14]$ & 0.004 & & - \\
\hline Hydroxychloroquine & $4.56[2.08-10.90]$ & 0.018 & & 0.002 \\
\hline Corticosteroids & 3.24 [1.22-8.98] & 0.001 & \multirow{2}{*}{$\begin{array}{l}5.91[2.10- \\
10.03]\end{array}$} & - \\
\hline $\begin{array}{l}\text { Time from symptoms onset to } 1 \mathrm{st} \\
\text { negative PCR }\end{array}$ & $1.39[1.20-1.65]$ & $\begin{array}{l}< \\
0.019\end{array}$ & & 0.017 \\
\hline Ventilator-acquired pneumoniae & 6.74 [2.85-15.09] & & \multirow{2}{*}{$\begin{array}{l}- \\
1.24[1.04- \\
1.50]\end{array}$} & 0.004 \\
\hline Bacteremia & $3.15[1.31-7.73]$ & 0.001 & & - \\
\hline SOFA score at Day 7 & $1.67[0.77-3.56]$ & 0.008 & \multirow{2}{*}{$\begin{array}{l}4.71[1.69- \\
14.41]\end{array}$} & - \\
\hline Acute kidney injury (KDIGO > 1) & & 0.022 & & \\
\hline Major bleeding & & $<.187$ & & \\
\hline Acute cor pulmonale & & & & \\
\hline
\end{tabular}

\section{Discussion}


This retrospective cohort study identified several risk factors for unfavorable outcome (death or requirement of invasive mechanical ventilation at 28 days after ICU admission) in critically ill adults in Toulouse who were hospitalized with Covid-19. In particular, chronic kidney disease on ICU admission, was associated with higher odds of in-hospital unfavorable outcome. Among follow-up data, acute kidney injury and ventilator- associated pneumonia were negatively related to patient's outcome. It is worth noting that in this cohort, data from repeated and detailed pathophysiological characterization of Covid-19 respiratory failure 22 - including respiratory system mechanics - was not associated to 28 days patient's outcome. Altogether these results, put the spotlight on the major impact of non-respiratory organ failures in this challenging clinical setting.

The case fatality rate of $15.3 \%$ in this cohort is lower to that reported among critically ill patients in Chinese $^{7}$, Italian ${ }^{6}$ or American ${ }^{5,23}$ hospitals. This data could reflect a different organization of health care system. In our case, the needed amount of intensive care support has been provided by a regional network of previously existing level 2 ICUs (providing invasive and noninvasive mechanical ventilation, renal replacement therapies). Public and private hospitals participating to this network, early canceled elective operations and speed home patients with less critical illness. Those efforts permitted to rapidly increase the number of ICU free beds available for coronavirus patients (rising from 119 to 235 over February, 2020).

Kidney involvement is frequent in Covid- $19 ;>40 \%$ of cases have abnormal proteinuria at hospital admission ${ }^{19}$. In line with previous reports ${ }^{18}$, we have identified that acute kidney injury was observed in approximately one third of critically ill patients from our cohort. An understanding of the pathophysiological and mechanisms that can explain such a high rate of acute kidney injury is emerging. Factors as volume depletion, ventilator-induced hemodynamic effects, cytokine burden and renal tropism of SARS-CoV-2, might be common triggers and worsening factors for AKI. To our knowledge, we demonstrated for the first time that acute kidney injury is a relevant marker of disease severity and a negative prognostic factor in terms of mortality for critically ill Covid-19 patients. This result emphasizes the need for an early recognition of kidney involvement in Covid-19 and pave the way for the use of preventive and therapeutic measures to limit subsequent acute kidney injury or progression to more severe stages as aiming to reduce morbidity and mortality.

Data on the risk of secondary bacterial pneumonia in critically ill Covid-19 patients are limited. Converging evidence suggest that ventilator- associated pneumonia might be a frequent and major complication for these patients. SARS-CoV-2 can cause immune dysregulation due to increased production and circulation of cytokines, leading to hyper-inflammation and defects in lymphoid function ${ }^{21}$. In addition, it is well known that this virus infects the ciliated cells in the alveoli, and therefore stop carrying out their airway clearance activity. Finally, iatrogenic factors, as immunotherapeutics use, may contribute to increase the incidence of ventilator- associated pneumonia in this setting ${ }^{24}$. A recent report on 52 critically ill adult patients with SARS-CoV-2 pneumonia described ventilator-associated pneumonia in $11 \%$ of cases $^{7}$. We confirm this result, but we observed greater $(60.7 \%)$ proportion of 
ventilator- associated pneumonia from a larger cohort of patients. In addition, we observed that ventilator- associated pneumonia clearly worsens patient's clinical condition and is significantly associated to unfavorable outcome. Future studies should focus on assessing the impact of preventing, identifying and treating early ventilator-associated associated pneumonia to increase the chances of successful treatment in patients with Covid-19.

We observed a sustained viral detection in throat samples in both favorable and unfavorable outcomes. Interestingly, duration of the viral shedding was significantly longer in patients with unfavorable outcome. In Influenza virus and SARS-CoV-2 infection, prolonged viral shedding was associated with fatal outcome ${ }^{25}$. We think that this might have implication for both patients' isolation decision making and guidance about the length of antiviral treatment.

Based on current evidence and experience, chest CT scan is largely used for screening and early diagnosis of Covid-19 in high-risk groups. We have specifically explored the potential association

between chest CT imaging findings and patient outcome. A blinded and standardized ${ }^{10-12}$ image analysis process shown that patients in our cohort have very extensive lung anomalies. However, it should be noted that radiological scores were not associated to patient's outcome. Further studies are needed to know whether CT is suitable for severity assessment and patient's prognostication in severe forms of Covid-19.

Our study has several limitations. First, the interpretation of our findings might be limited by the sample size. It is possible that critically ill patients with established goals of care that were not consistent with admission to an ICU were not included in this study. However, by including all adult patients who were admitted for Covid-19 in the 12 ICUs of our network, we believe that our study population is representative of cases diagnosed and treated in Toulouse region. Second, despite the fact that this is a retrospective study, we think that a strength of this study is that it includes a homogenous population of critically ill adults with a lung insult from a well-defined cause. In addition, we have validated the information collected through repeated direct contacts with corresponding physician in each ICU.

\section{Conclusion}

To the extent of our knowledge, this is the first retrospective cohort study among critically ill adult patients with Covid-19, who have experienced a definite outcome. We found that acute kidney injury, but not physiopathological respiratory parameters, was associated with death or requirement of invasive mechanical ventilation at 28 days from ICU admission. Among ICU-related complication, ventilatorassociated pneumonia was a significant risk factor of unfavorable outcome. While waiting for effective SARS-CoV-2 antiviral treatments, prevention, early detection and care of these factors, might have a significant impact on critically ill Covid-19 patient's outcome.

\section{Declarations}


The ethical committee of the University Hospital of Toulouse approved the study and waived the requirement for informed consent.

\section{Constent for publication}

All authors have provided final approval of this version to be published

\section{Availability of supporting data}

The authors confirm that the data supporting the findgins of this study are available within the article and its supplementary material. The whole dataset supporting the findings of this study are available from the corresponding author, upon reasonable request.

\section{Competing interests}

The authors report no competing interests related to the manuscript.

\section{Funding}

This work was supported by Grant from University Hospital of Toulouse. The sponsor of the study had no role in study design, data collection, data analysis, data interpretation, or writing of the report.

\section{Author's contributions}

SS takes the responsibility for the content of the manuscript, including the data and analysis. He conceived the study and has personally made contributions to the design of the study, the acquisition of data, and the analysis and interpretation of data. WB, BS, CZ, JI, SC, DG have substantially participated at the data acquisition. DG has made contribution to the design of the study. TF, SS and BB have contributed to the conception and analysis of data. SS and DG wrote the report. All authors have revised the submitted article critically for important intellectual content and they have provided final approval of this version to be published. All authors agree to the conditions outlined in the Guide for Authors.

\section{Acknowledgements}

The authors thank the medical and nurse staff of the 12 ICUs from the "COVID-19 Toulouse ICU Network" for their commitment and active participation to the study. We would like that the names of the individual members of the group "COVID-19 Toulouse ICU Network" to be searchable throught their individual PubMed records.

\section{References}


1. Guan WJ, Ni ZY, Hu Y, et al. Clinical Characteristics of Coronavirus Disease 2019 in China. N Engl J Med. 2020;382(18):1708-20.

2. Huang $C$, Wang $Y, L i X$, et al. Clinical features of patients infected with 2019 novel coronavirus in Wuhan, China. Lancet. 2020;395(10223):497-506.

3. Lipsitch M, Swerdlow DL, Finelli L. Defining the Epidemiology of Covid-19 - Studies Needed. N Engl J Med. 2020;382(13):1194-6.

4. Ruan Q, Yang K, Wang W, Jiang L, Song J. Clinical predictors of mortality due to COVID-19 based on an analysis of data of 150 patients from Wuhan, China. Intensive Care Med. 2020;46(5):846-8.

5. Bhatraju PK, Ghassemieh BJ, Nichols M, et al. Covid-19 in Critically III Patients in the Seattle Region Case Series. N Engl J Med. 2020;382(21):2012-22.

6. Grasselli G, Zangrillo A, Zanella A, et al. Baseline Characteristics and Outcomes of 1591 Patients Infected With SARS-CoV-2 Admitted to ICUs of the Lombardy Region, Italy. JAMA. 2020.

7. Yang $X, Y u Y, X u$ J, et al. Clinical course and outcomes of critically ill patients with SARS-CoV-2 pneumonia in Wuhan, China: a single-centered, retrospective, observational study. Lancet Respir Med. 2020;8(5):475-81.

8. Cordes AK, Heim A. Rapid random access detection of the novel SARS-coronavirus-2 (SARS-CoV-2, previously 2019-nCoV) using an open access protocol for the Panther Fusion. J Clin Virol. 2020;125:104305.

9. WHO. Clinical management of severe aute respiratory infection when novel coronavirus (nCoV) infection is suspected: interim guidence, 25 januray 2020.

10. Shi H, Han X, Jiang N, et al. Radiological findings from 81 patients with COVID-19 pneumonia in Wuhan, China: a descriptive study. Lancet Infect Dis. 2020;20(4):425-34.

11. Zhou Z, Guo D, Li C, et al. Coronavirus disease 2019: initial chest CT findings. Eur Radiol. 2020.

12. Li K, Fang Y, Li W, et al. CT image visual quantitative evaluation and clinical classification of coronavirus disease (COVID-19). Eur Radiol. 2020.

13. Kalil AC, Metersky ML, Klompas M, et al. Management of Adults With Hospital-acquired and Ventilator-associated Pneumonia: 2016 Clinical Practice Guidelines by the Infectious Diseases Society of America and the American Thoracic Society. Clin Infect Dis. 2016;63(5):e61-111.

14. Khwaja A. KDIGO clinical practice guidelines for acute kidney injury. Nephron Clin Pract. 2012;120(4):c179-184.

15. Shi S, Qin M, Shen B, et al. Association of Cardiac Injury With Mortality in Hospitalized Patients With COVID-19 in Wuhan, China. JAMA Cardiol. 2020.

16. Mekontso Dessap A, Boissier F, Charron C, et al. Acute cor pulmonale during protective ventilation for acute respiratory distress syndrome: prevalence, predictors, and clinical impact. Intensive Care Med. 2016;42(5):862-70.

17. Arabi YM, Balkhy HH, Hayden FG, et al. Middle East Respiratory Syndrome. N Engl J Med. 2017;376(6):584-94. 
18. Ronco C, Reis T, Husain-Syed F. Management of acute kidney injury in patients with COVID-19. Lancet Respir Med. 2020.

19. Cheng $Y$, Luo R, Wang K, et al. Kidney disease is associated with in-hospital death of patients with COVID-19. Kidney Int. 2020;97(5):829-38.

20. Henderson WR, Chen L, Amato MBP, Brochard LJ. Fifty Years of Research in ARDS. Respiratory Mechanics in Acute Respiratory Distress Syndrome. Am J Respir Crit Care Med. 2017;196(7):822-33.

21. Cao X. COVID-19: immunopathology and its implications for therapy. Nat Rev Immunol. 2020;20(5):269-70.

22. Ziehr DR, Alladina J, Petri CR, et al. Respiratory Pathophysiology of Mechanically Ventilated Patients with COVID-19: A Cohort Study. Am J Respir Crit Care Med. 2020.

23. Richardson S, Hirsch JS, Narasimhan M, et al. Presenting Characteristics, Comorbidities, and Outcomes Among 5700 Patients Hospitalized With COVID-19 in the New York City Area. JAMA. 2020.

24. Brun-Buisson C, Richard JC, Mercat A, Thiebaut AC, Brochard L, Group R-SAHNvR. Early corticosteroids in severe influenza $\mathrm{A} / \mathrm{H} 1 \mathrm{~N} 1$ pneumonia and acute respiratory distress syndrome. Am J Respir Crit Care Med. 2011;183(9):1200-6.

25. Wang Y, Guo Q, Yan Z, et al. Factors Associated With Prolonged Viral Shedding in Patients With Avian Influenza A(H7N9) Virus Infection. J Infect Dis. 2018;217(11):1708-17.

\section{Figures}




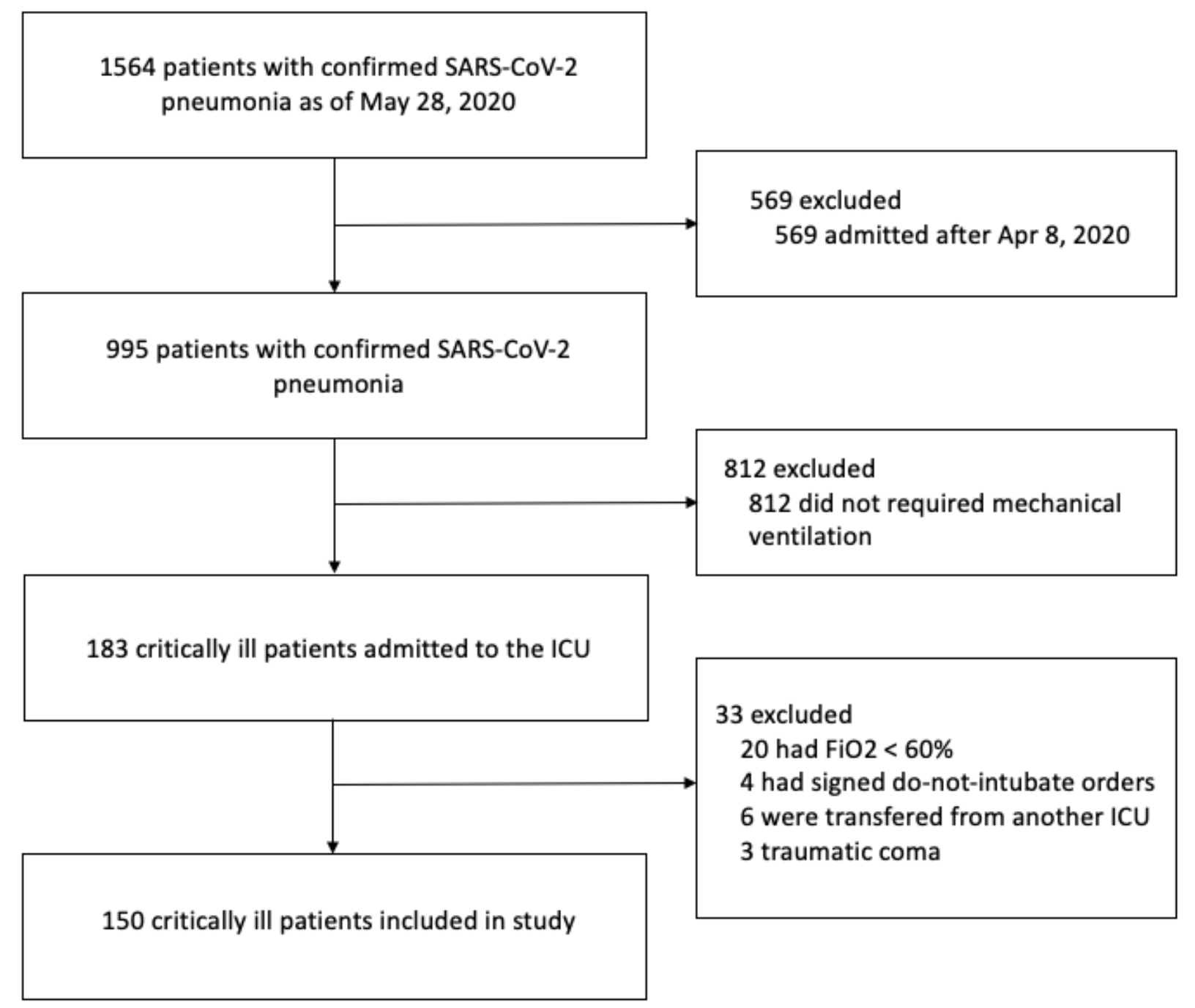

Figure 1

Flow chart. 6 patients were transfered from another French region. All these patients required mechanical ventilation before their transfer, and therefore were not included in the current study. 3 patients were admitted from severe traumatic brain injury, requiring invasive mechanical ventilation. Despite the fact that SARS-CoV-2 was detected in the naso-pharyngeal swab of these patients, none of them were not included in the study. 


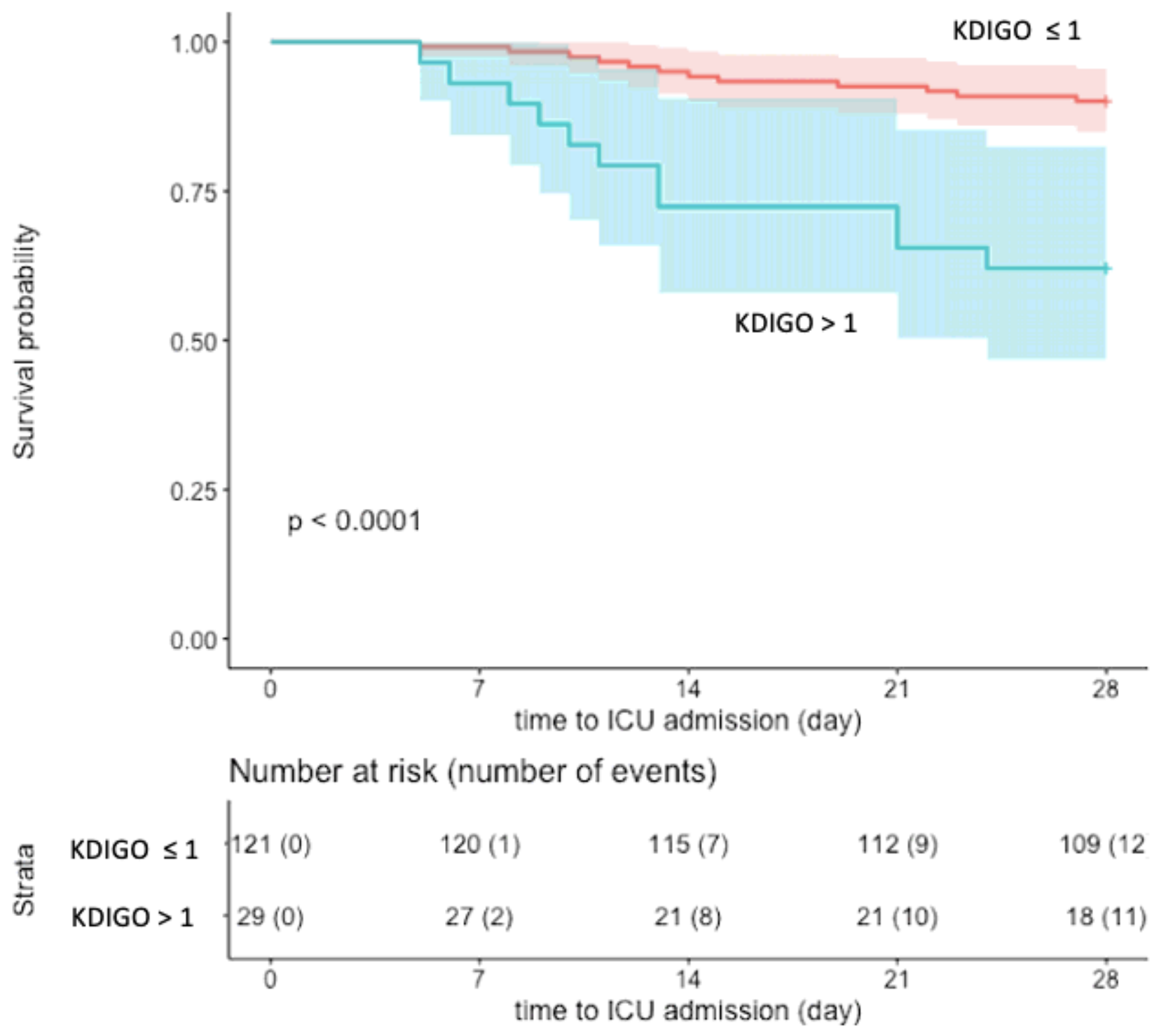

Figure 2

Survival probability over time and acute kidney injury. KDIGO score was binarized (KDIGO = or $<1$ vs. $\mathrm{KDIGO}>1$ ) to assess the link between acute kidney injury and mortality 28 days after ICU admission. Abbreviation: KDIGO = Kidney Disease Improving Global Outcomes score.

\section{Supplementary Files}

This is a list of supplementary files associated with this preprint. Click to download.

- eTable1.docx

- eFigure2.docx

- eFigure1.tiff

- eAppendix1.docx 